Tecno Lógicas

ISSN 0123-7799

Vol. 18, No. 35, pp. 93-104

Julio-diciembre de 2015

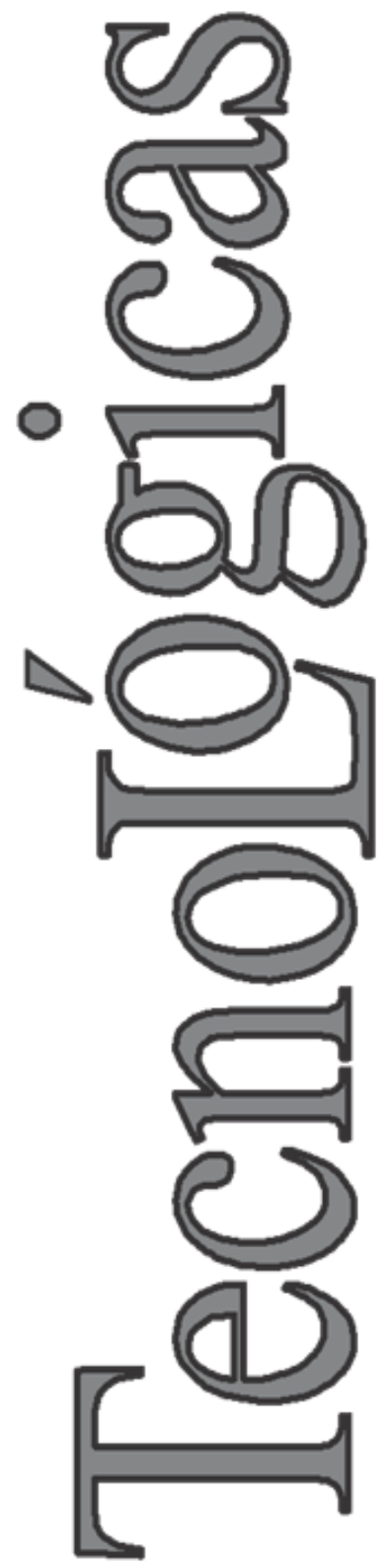

Copyright 2015 por autores y Tecno Lógicas Este trabajo está licenciado bajo una Licencia Internacional Creative

Commons Atribución (CC BY)

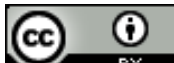

\section{Análisis de las correlaciones existentes del ángulo de fricción efectivo para suelos del piedemonte oriental de Bogotá usando ensayos in situ}

\section{Analysis of the existing correlations of effective friction angle for eastern piedmont soils of Bogota from in situ tests}

July E. Carmona-Álvarez ${ }^{1}$ y Juan C. Ruge-Cárdenas²

Recibido: 7 de enero de 2015,

Aceptado: 18 de junio de 2015

Cómo citar / How to cite

J. E. Carmona-Álvarez y J. C. Ruge-Cárdenas, "Análisis de las correlaciones existentes del ángulo de fricción efectivo para suelos del piedemonte oriental de Bogotá usando ensayos in situ", Tecno Lógicas, vol. 18 , no. 35 , pp. 93-104, 2015.

1 Ingeniera civil, Programa de Ingeniería Civil, Facultad de Ingeniería, Universidad Católica de Colombia, Bogotá-Colombia, jecarmona91@gmail.com

2 PhD. en Geotecnia, Especialización en Ingeniería de Pavimentos, Facultad de Ingeniería, Universidad Católica de Colombia, BogotáColombia,jcruge@ucatolica.edu.co 


\section{Resumen}

Estimar el ángulo de fricción efectivo del suelo a partir de ensayos in situ es una tarea complicada debido a las altas tasas de deformación existentes en este tipo de ensayos, que tienden a ser demasiado invasivos y perturban las vecindades de la profundidad del ensayo y aún la muestra que eventualmente se toma en el sitio. Así mismo, la mayoría de correlaciones encontradas en la literatura actual, para obtener el ángulo de fricción efectivo, usando ensayos de campo han sido desarrolladas para suelos de otras latitudes, por tal razón al ser implementadas en suelos tropicales presentan alta dispersión, cuando se desea comparar los valores de parámetros de campo, con los resultados reales obtenidos en el laboratorio. Esta investigación busca mediante el uso de ensayos in situ, definir por medio de análisis de diferentes correlaciones, cuál de estas se ajusta mejor a las condiciones propias de suelos del piedemonte de la ciudad de Bogotá, la cual se considera la problemática primordial a resolver en el artículo. Para el presente estudio serán utilizados datos provenientes del SPT - Standard Penetration Test (de amplio uso en Colombia) y SPT-T Standard Penetration Test + Torque (nunca antes llevado a cabo en el país), realizados teniendo en cuenta la normativa acorde para cada ensayo. Considerando que para el SPT-T no existe norma local que rija este tipo de ensayos. Las correcciones por procedimientos de campo de los ensayos que se implementaron fueron realizadas por confinamiento efectivo y transferencia de energía del martillo golpeador, ya que en el estado del arte del tema son definidos como los que influyen de manera directa en la confiabilidad de los resultados. Los resultados finales muestran la tendencia de cada una de las metodologías usadas para obtener la correlación, en relación con el valor real del ángulo de fricción efectivo, obtenido de ensayos de laboratorio.

\section{Palabras clave}

Ensayo de penetración estándar SPT, SPT-T, ensayos in situ, correlación de parámetros geotécnicos, ángulo de fricción efectivo.

\section{Abstract}

To estimate the effective friction angle of soil from in situ test is a complicated job, due to high rates of strain existing in this kind of tests, which tend to be too invasive and disturb the vicinities of test depth, even the sample that eventually is taken at the site. Likewise, the most of the correlations found in the current bibliography to obtain the effective friction angle using field tests, have been developed for soils from different regions. For that reason when are implemented on tropical soils present high scatter, to compare the field parameter values with real results obtained at the lab. This research aims to use in situ tests define through of analysis of different correlations, which fits adequately to the specific conditions of the piedmont soils of Bogota. For the present study will be utilized data from SPT (widely used in Colombia) and SPT-T (never before conducted in the country), carried out considering the appropriated norms to each test, taking in account to SPT-T, doesn't exist local standard governing such tests. The correlations for field procedures of the tests implemented were for effective confining and energy transference of the SPT hammer, since the state-of-the-art mentions it as the most affect the reliability of the final results. The final results show the tendency of the methodologies used to obtain the correlation, in relation with the real value of effective friction angle from of lab tests.

\section{Keywords}

SPT, SPT-T, in situ tests, correlation of geotechnical parameters, effective friction angle. 


\section{INTRODUCCIÓN}

Desde comienzos del siglo 20 cuando se originó el uso del SPT, se identificó que era un ensayo extremadamente útil y versátil para determinar la resistencia de un suelo. En gran medida, gracias a los trabajos de H. Mohr y G. Fletcher en 1927, cuando intentaron normalizar el ensayo al hincar un muestreador de $50 \mathrm{~mm}$ de diámetro, usando un martillo de $62,5 \mathrm{~kg}$, transfiriendo una energía potencial alcanzada desde una altura de impacto de $760 \mathrm{~mm}$. El ensayo originalmente fue diseñado para evaluar la capacidad portante de cimentaciones superficiales en suelos arenosos, justamente a la fecha, la correlación más aceptada es la correspondiente al ángulo de fricción efectivo y densidad relativa del suelo, aunque existen diferentes ecuaciones para el cálculo de diversos parámetros geotécnicos, las cuales se consideran poco confiables.

Terzaghi \& Peck [1] acuñaron el término "ensayo de penetración estándar", y presentaron la primera correlación entre el número de golpes $\left(N_{S P T}\right)$ y la densidad relativa, finalmente en 1967 fue estandarizado por la ASTM. Sin embargo, en la actualidad ha experimentado diferentes reformas de acuerdo con el procedimiento de ejecución, particularmente la debida a la transferencia de energía generada por el impacto del martillo al muestreador a través de las barras, entre otras (Bowles [2]):

1. Diferente fabricación de los equipos.

2. Diversas configuraciones del martillo de golpes, de las cuales tres son las más comunes: antiguo de pesa con varilla de guía interna, martillo anular donut (ver Fig. 1), y de seguridad.

3. Control de la altura de golpeo: si es manual, la manera como se controla la altura; y si es con la cuerda en la polea del equipo, depende de: el diámetro y condición de la cuerda y la polea, del número de vueltas de la cuerda en la polea y de la altura real de caída del martillo.

4. Uso de revestimiento interno en el muestreador (normalmente no es usado).

5. Longitud de la barra desde el sitio de golpeo y el muestreador.

6. Diámetro de la perforación.

7. La presión de confinamiento efectiva al muestreador, la cual depende del esfuerzo vertical en el sitio del ensayo.

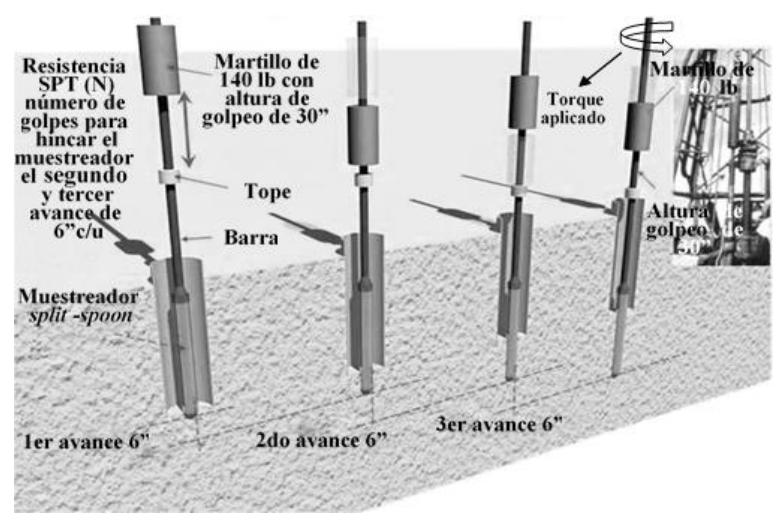

Fig. 1. Procedimiento de ensayos de penetración estándar y aplicación posterior de torque. Fuente: Modificado de [3], cortesía de K. Tawfiq, Florida State University, USA

Desde la invención del ensayo hasta hoy día, la prueba de campo ha sido foco de estudio para grandes investigadores de estudios geotécnicos como Terzaghi \& Peck [1], Seed \& Idris [4], Meyerhof [5], Skempton [6], entre otros, quienes se basaron en métodos diseñados desde el año 1927, dando como resultado correlaciones provenientes del parámetro SPT obtenido $\left(N_{S P T}\right.$ o $\left.N_{F}\right)$.

En Colombia, González [7] y Montenegro [8] han hecho el aporte más significativo sobre ensayos de penetración estándar, suministrando a las investigaciones al respecto una ecuación logarítmica para calcular la corrección de la presión de confinamiento y una correlación para encontrar el ángulo de fricción, las cuales en principio se ajustan apropiadamente de manera general a las condiciones en cómo se articula el ensayo con las características del suelo local. 
De otra parte, el SPT-T (Standard Penetration Test + Torque), posee la ventaja de combinar perfectamente los esquemas de los ensayos clásicos SPT (Standard Penetration Test) y VST (Vane Shear Test). Fue llevado a cabo por primera vez por Ranzini [9], quien propuso una leve alteración en el procedimiento del SPT basado en la observación en el momento de finalizar el ensayo, cuando el operador aplica una torsión a la barra con una herramienta manual (más detalles en Ruge et al. [10], Ruge y Da Cunha [11]).

Esencialmente usa en principio el mismo procedimiento del ensayo SPT (NBR 6484/80 e INV-111-2012), al penetrarse un muestreador split-spoon en el geomaterial bajo análisis, posteriormente es realizada una rotación en el muestreador por medio de un elemento en el nivel del terreno llamado torquímetro, el cual mide la torsión máxima suficiente para vencer la resistencia opuesta por el suelo, y de esta manera obtener la fricción lateral del tándem muestreador-suelo (ver Fig. 2); también es medido el torque residual en el suelo [12].

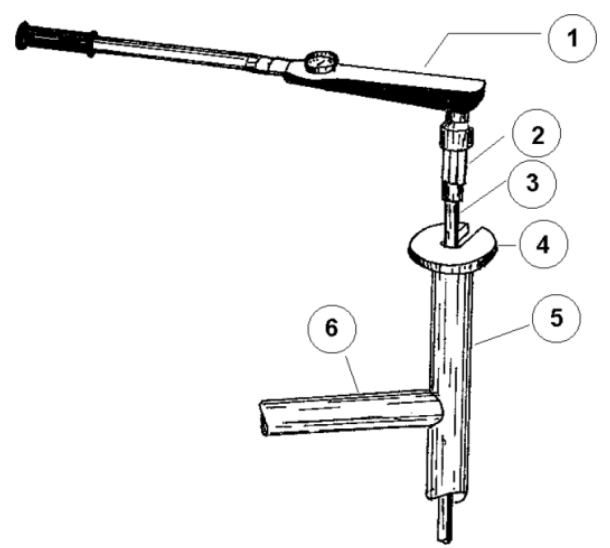

Fig. 2. Torquímetro y sus partes. Fuente: [13]

Pretendiendo resolver la problemática planteada en la motivación y lograr determinar la estratigrafía del suelo de la carrera $7^{\mathrm{a}}$ entre calle 38 y calle 170 , se tomaron los ensayos SPT realizados para el diseño de las estaciones del sistema de transporte masivo (TransMilenio) [14] y SPT-T realizados en el campo experimental de la Uni- versidad Católica de Colombia, para calcular las correcciones de la presión de confinamiento y energía de transferencia del martillo, con los cuales se determinaron correlaciones con el ángulo de fricción calculado.

Es bien conocido en el ámbito geotécnico (incluso las discusiones sobre este tema datan prácticamente desde la popularización del ensayo), que el SPT no es igual de confiable para la evaluación de materiales arcillosos, ya que las presiones de poro generadas en suelos poco permeables, la adhesión muestreador-suelo (poco estudiada hasta la fecha) y la perturbación causada por la naturaleza dinámica del ensayo, hacen que los resultados, por lo menos en la obtención de parámetros no drenados, no sea la más correcta; aunque la estimación tienda a ser conservadora.

Sin embargo, cuando se tiene una experiencia local y conocimiento del suelo estudiado, como es el caso del piedemonte oriental, se pueden avalar los resultados obtenidos mediante la comparación con los parámetros obtenidos del laboratorio, la cual es una de las fases del estudio acá presentado.

\section{METOdOLOGía}

Teniendo como insumo de la investigación las perforaciones usadas para el estudio geotécnico del sistema de transporte masivo para la carrera séptima de Bogotá [13], la cual es un corredor que se alinea sobre depósitos de piedemonte de los cerros orientales, es decir conos de deyección constituidos por geomateriales que han sido transportados por la gravedad y localizados en forma de abanico, donde predominan los materiales granulares gruesos con matriz arcillosa, según la microzonificación sísmica de Bogotá [15], se analizaron la totalidad de los mencionados sondeos, los cuales incluyen datos de ensayos de campo y laboratorio. 
Fueron estudiadas cerca de 100 perforaciones entre 5 y $20 \mathrm{~m}$ de profundidad cada una, las cuales fueron situadas aproximadamente cada $500 \mathrm{~m}$ desde la calle 34 hasta la calle 170, donde se consideraron los parámetros del ensayo de penetración estándar, para realizar la corrección por confinamiento de cada estrado ensayado, según las siguientes metodologías de la Tabla 1 , donde el valor $R s$ corresponde a la relación entre el esfuerzo vertical efectivo y la presión atmosférica, que estandariza la corrección a un esfuerzo vertical de referencia.

Tabla 1. Metodologías para corregir el confinamiento en el SPT. Fuente: Modificado de [7]

\begin{tabular}{lcc}
\hline \multicolumn{1}{c}{ Autor } & Año & Ecuación \\
\hline Terzaghi \& Peck [1] & 1948 & $C_{N}=\frac{\log (20 / R s)}{\log (20)}$ \\
Meyerhof [5] & 1975 & $C_{N}=\frac{1,7}{0,7+R s}$ \\
Seed \& Idriss [4] & 1983 & $C_{N}=1-\mathrm{K} \log (R s)$ \\
Schmertmann [16] & 1983 & $C_{N}=\frac{32,5}{10,2+20,3 R s}$ \\
Seed [17] & 1985 & $C_{N}=1-1,25 \log (R s)$ \\
Liao \& Whitman [18] & 1986 & $C_{N}=\sqrt{1 / R s}$ \\
Skempton [6] & 1986 & $C_{N}=\frac{2}{1+R s}$ \\
González [7] & 1999 & $C_{N}=\log (10 / R s)$ \\
\hline
\end{tabular}

Después de realizada la corrección por confinamiento $\left(C_{N}\right)$, la cual afecta de manera directamente proporcional el valor del número de golpes del SPT en campo $\left(N_{F}\right)$, el valor obtenido para el número de golpes corregidos se denota con $\left(N_{1}\right)$. A partir de este último dato obtenido, se correlaciona el ángulo de fricción efectivo usando las propuestas de los siguientes autores (ver Tabla 2), también para cada uno de los ensayos en las 100 perforaciones analizadas.

Usando las recomendaciones de González [7] se realiza una corrección por energía, de acuerdo con los procedimientos normalmente usados en el país, donde se usa martillo de golpeo tipo donut, por lo cual se tomó de manera conservativa la eficiencia de transmisión de energía de $45 \%$ (valor E de la Tabla 3), del martillo de golpeo hasta el muestreador en el extremo de la perforación (ver Tabla 3).

Tabla 2. Correlaciones para obtener el ángulo de fricción efectivo a partir del $N_{1}$. Fuente: [7]

\begin{tabular}{|c|c|c|}
\hline Autor & Año & Ecuación \\
\hline Terzaghi \& Peck [1] & 1948 & $\phi^{\prime}=28,5+0,25 N_{1}$ \\
\hline Peck et al. [19] & 1953 & $\phi^{\prime}=26,25\left(2-\left(e^{\frac{-N_{1}}{62}}\right)\right)$ \\
\hline Kishida [20] & 1969 & $\phi^{\prime}=15+\sqrt{20 N_{1}}$ \\
\hline Schmertmann [16] & 1970 & $\phi^{\prime}=\tan ^{-1}\left[\left(\frac{N_{1}}{32,5}\right)^{0,34}\right]$ \\
\hline $\begin{array}{l}\text { JNR (Japan National } \\
\text { Railway) [21] }\end{array}$ & 1999 & $\phi^{\prime}=27+0,30 N_{1}$ \\
\hline $\begin{array}{l}\text { JRB (Japan Road Bu- } \\
\text { reau) [21] }\end{array}$ & 1986 & $\phi^{\prime}=15+\sqrt{9,375 N_{1}}$ \\
\hline Hatanaka \& Uchida [22] & 1996 & $\phi^{\prime}=\sqrt{20 N_{1}}+20$ \\
\hline $\begin{array}{l}\text { Montenegro \& Gonzalez } \\
\text { [8] }\end{array}$ & 2014 & $\phi^{\prime}=12,79+\sqrt{25,86 N_{1}}$ \\
\hline
\end{tabular}

De acuerdo con la Tabla 3 y la metodología de [7], los valores para la correlación del ángulo de fricción efectivo transformadas para una energía del $45 \%$, debido precisamente a que el ensayo en Colombia sigue realizándose con martillos de impacto tipo donut, y algunas correlaciones como las japonesas consideran en sus ensayos transferencias de energía más altas, por lo cual deben ser transformadas al contexto colombiano $(\mathrm{E}=45 \%)$. Estas fueron definidas de la forma presentada en la Tabla 4.

Referente al SPT-T se evaluará la relación $T / N$ del ensayo, considerándose los datos recolectados del campo experimental situado dentro del piedemonte de los cerros orientales descritos anteriormente, los análisis toman en cuenta la caracterización del suelo y la geología local. 
Análisis de las correlaciones existentes del ángulo de fricción efectivo para suelos del piedemonte oriental de Bogotá usando ensayos in situ

Tabla 3. Eficiencias promedio y corrección para energía $-\mathrm{E}_{\mathrm{ref}}=60 \%$. Fuente: $[23]$

\begin{tabular}{|c|c|c|c|c|c|}
\hline \multirow{2}{*}{ País } & \multicolumn{2}{|c|}{ Martillo de golpeo } & \multirow{2}{*}{ E (\%) } & \multirow{2}{*}{$\begin{array}{c}\mathrm{C} \text { (factor } \\
\text { multiplicador) }\end{array}$} & \multirow{2}{*}{ Referencia } \\
\hline & Tipo & Accionamiento & & & \\
\hline Argentina & Donut & Polea-soga & 45 & 0,75 & Seed et al [17] \\
\hline Brasil & Guía interna & Manual & 72 & 1,2 & Décourt [23] \\
\hline China & Donut & Caída libre & 60 & 1,0 & Seed et al [17] \\
\hline Colombia & Donut & Polea-soga & 45 & 0,75 & González [7] \\
\hline \multirow{2}{*}{ Japón } & Donut & Caída libre & 85 & 1,40 & \multirow{2}{*}{ Riggs [24] } \\
\hline & Donut & Polea-soga & 68 & 1,13 & \\
\hline Paraguay & Guía interna & Manual & 72 & 1,20 & -..-....- \\
\hline \multirow{2}{*}{ Reino Unido } & Donut & Caída libre & 60 & 1,0 & \multirow{2}{*}{ Skempton [6] } \\
\hline & Donut & Polea-soga & 50 & 0,83 & \\
\hline \multirow{3}{*}{ EUA } & Donut & Polea-soga & 45 & 0,75 & Seed et al [17] \\
\hline & Seguridad & Polea-soga & 60 & 1,0 & Seed et al [17] \\
\hline & Seguridad & Caída libre & 85 & 1,40 & Riggs [24] \\
\hline Venezuela & Donut & Polea-soga & 43 & 0,72 & -..-..-- \\
\hline
\end{tabular}

Tabla 4. Transformaciones del ángulo de fricción efectivo según la energía trasferida. Fuente: [7]

\begin{tabular}{lccc}
\hline \multicolumn{1}{c}{ Autor } & Año & Ecuación & Transformación \\
\hline Terzaghi \& Peck [1] & 1948 & $\phi^{\prime}=28,5+0,25 N_{1_{45}}$ & $\phi_{45}^{\prime}=28,5+0,25 N_{1_{45}}$ \\
Peck et al. [19] & 1953 & $\phi^{\prime}=26,25\left(2-\left(e^{\frac{-N_{1_{45}}}{62}}\right)\right)$ & $\phi_{45}^{\prime}=26,25\left(2-\left(e^{\left.\left.\frac{-N_{1_{45}}}{62}\right)\right)}\right.\right.$ \\
Kishida [20] & 1969 & $\phi^{\prime}=15+\sqrt{20 N_{1_{72}}}$ & $\phi_{45}^{\prime}=15+\sqrt{12,5 N_{1_{45}}}$ \\
Schmertmann [16] & 1970 & $\phi^{\prime}=\tan ^{-1}\left[\left(\frac{N_{1_{60}}}{32,5}\right)^{0,34}\right.$ & $\phi_{45}^{\prime}=\tan ^{-1}\left[\left(\frac{N_{1_{45}}}{43,3}\right)\right.$ \\
JNR (Japan National Railway) [21] & 1999 & $\phi^{\prime}=27+0,30 N_{1_{72}}$ & $\phi^{\prime}{ }_{45}=27+0,1875 N_{1_{45}}$ \\
JRB (Japan Road Bureau) [21] & 1986 & $\phi^{\prime}=15+\sqrt{15 N_{1_{72}}}$ & $\phi^{\prime}{ }_{45}=15+\sqrt{9,375 N_{1_{45}}}$ \\
Hatanaka \& Uchida [22] & 1996 & $\phi^{\prime}=\sqrt{20 N_{172}}+20$ & $\phi_{45}^{\prime}=\sqrt{12,5 N_{1_{45}}}+20$ \\
Montenegro \& González [8] & 2014 & $\phi^{\prime}=12,79+\sqrt{25,86 N_{1_{72}}}$ & $\phi_{45}^{\prime}=12,79+\sqrt{16,16 N_{1_{45}}}$ \\
\hline
\end{tabular}

\section{RESULTADOS Y DISCUSIÓN}

Aunque los suelos de piedemonte de los cerros orientales se consideran geológicamente afines, desde el punto de vista geotécnico al observar la estratigrafía detallada de cada una de los sitios escogidos, se decidió zonificar en dos partes el corredor correspondiente a estos conos de deyección: suelos arcillosos con contenido de limos y suelos arcillosos con contenido de limos y materiales de relleno.
Para la primera zona geotécnica se seleccionaron las perforaciones marcadas en la Fig. 3, en el corredor bajo estudio. En la Fig. 4 se puede observar la evolución de la corrección por confinamiento, en función del esfuerzo efectivo vertical, para las perforaciones correspondientes a la calle 38 , para cada una de las propuestas enunciadas. Es importante observar que esta corrección tiende a aumentar el número de golpes en campo para esfuerzos efectivos menores. 
La tendencia de todas las correcciones presenta un comportamiento similar y es razonable plantear una curva promedio para el análisis, de acuerdo con la metodología propuesta por [8], que recomienda este tipo de análisis simple. No obstante la corrección logarítmica definida para suelos tropicales, presenta una tendencia que se ajusta adecuadamente al promedio [7].

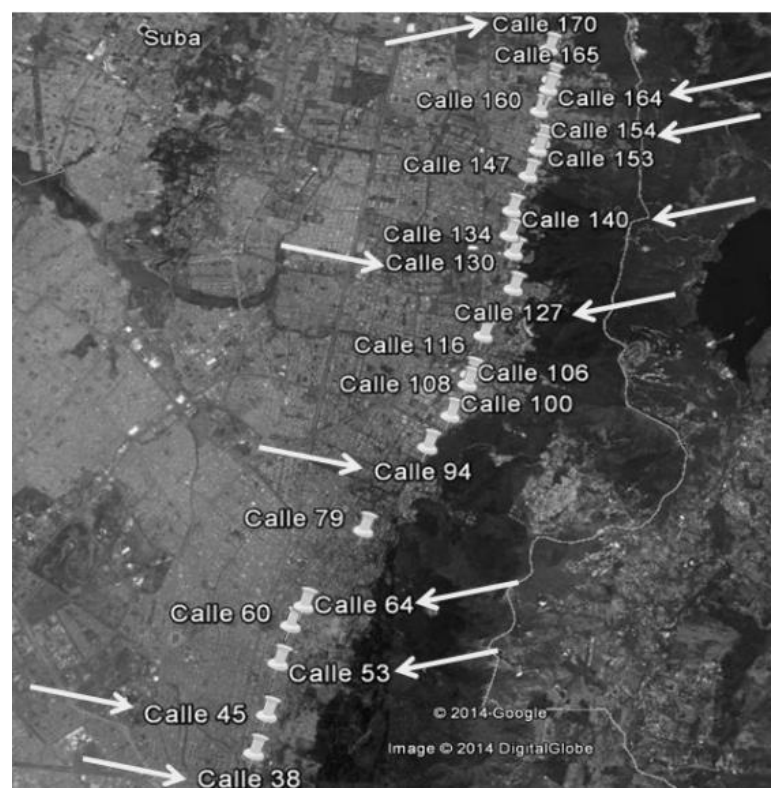

Fig. 3. Sitios de perforaciones para la primera zona geotécnica Fuente: Modificado de Google Earth.

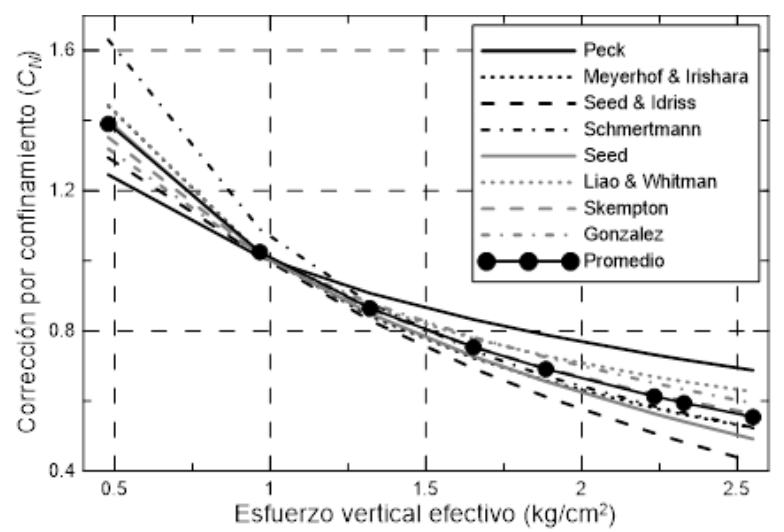

Fig. 4. Corrección por confinamiento para sondeos cercanos a la calle 38 correspondiente a la primera zona geotécnica. Fuente: Autores

Para obtener los valores de los ángulos de fricción correlacionados de las ecuaciones enunciadas en la Tabla 4, se consideró la profundidad de la muestra usada para obtener este parámetro del corte directo
$(1,00-4,75 \mathrm{~m})$, y de acuerdo con esto se realizó un promedio de la corrección por confinamiento a una profundidad de $2,3 \mathrm{~m}$, para cada uno de los autores ya mencionados, afectada de igual forma por la corrección de energía.

En este caso, para los sondeos de la calle 38 se muestran en la Tabla 5 estos valores, a priori la propuesta de Peck et al. [19], Hatanaka \& Uchida [22] y JNR [21] revelan un valor cercano al obtenido de la muestra en laboratorio, sin embargo, las otras metodologías también se encuentran próximas al valor real $\phi^{\prime}$ lab, excepto Kishida [20], JRB [21] y Montenegro \& González [8], las cuales son las más conservadoras de las propuestas analizadas. El valor de laboratorio del ángulo de fricción efectivo fue obtenido mediante el ensayo de corte directo drenado, revelando unos valores bajos de cohesión menores a $10 \mathrm{kN} / \mathrm{m}^{2}$.

Se esperaba que la propuesta de [8] tuviera un menor error de dispersión teórico, respecto al valor de laboratorio, sin embargo, vale la pena aclarar que esta metodología fue obtenida sobre depósitos aluviales conformados en llanuras, abanicos y terrazas, característicos de los suelos llaneros de Colombia, diferentes a los aquí estudiados.

Tabla 5. Correlaciones del ángulo de fricción para la primera zona geotécnica escogida

(Zona I; $\left.\overline{C_{N}}=1,236 ; \mathrm{N}_{1}=6 ; \phi^{\prime} l a b=28,5^{\circ}\right)$. Fuente: Autores

\begin{tabular}{lcc}
\hline \multicolumn{1}{c}{ Autor } & $\phi^{\prime}$ & $\begin{array}{c}\text { Error de disper- } \\
\text { sión teórico }\end{array}$ \\
\hline Terzaghi \& Peck [1] & $30,05^{\circ}$ & $+5,4 \%$ \\
Peck et al. [19] & $28,74^{\circ}$ & $+0,8 \%$ \\
Kishida [20] & $23,79^{\circ}$ & $-17,6 \%$ \\
Schmertmann [16] & $29,49^{\circ}$ & $+3,5 \%$ \\
JNR [21] & $28,16^{\circ}$ & $-1,2 \%$ \\
JRB [21] & $22,61^{\circ}$ & $-20,7 \%$ \\
Hatanaka \& Uchida [22] & $28,79^{\circ}$ & $+1,0 \%$ \\
Montenegro \& González [8] & $22,78^{\circ}$ & $-20,0 \%$ \\
\hline
\end{tabular}

Para la segunda zona geotécnica definida se muestra en la Fig. 5 la localización de los sitios analizados. En este documento se mostrarán los resultados provenientes de 
la calle 60 (suelos arcillosos con contenido de limos y materiales de relleno).

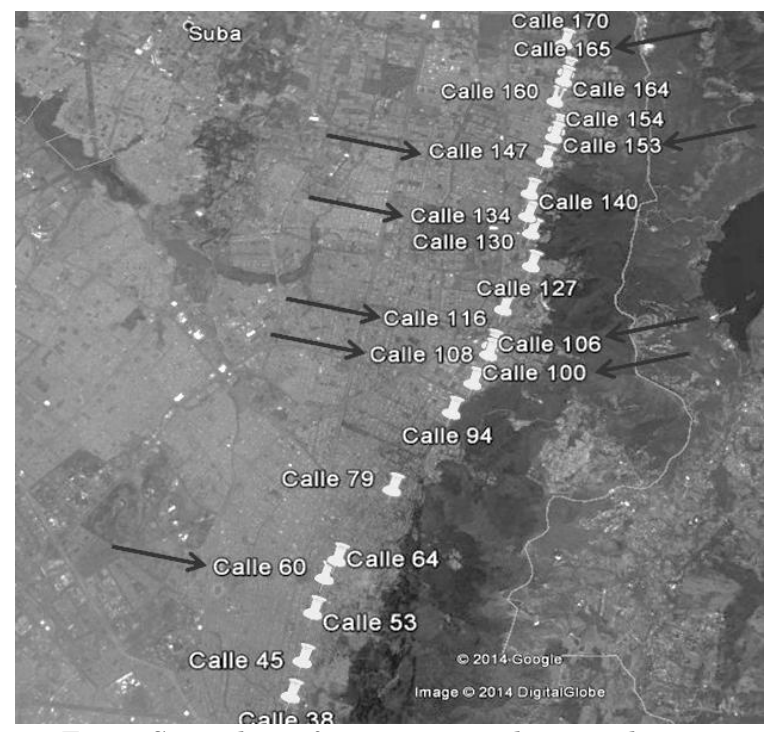

Fig. 5. Sitios de perforaciones para la segunda zona geotécnica. Fuente: Modificado de Google earth.

En la Fig. 6 se observa de igual forma la influencia de la profundidad sobre la corrección por confinamiento para cada uno de los autores mostrados, en este caso para profundidades más altas los valores de $C_{N}$ tienden a unificarse cerca de $0,9 \mathrm{y}$ para valores superficiales el rango de diferencia es relativamente pequeño. Las metodologías de Seed \& Idriss [4], Skempton [6] y González [7], siguen siendo las que se ajustan de mejor modo al promedio del comportamiento de todas las curvas, al igual que en la Fig. 4, sin embargo, se continúa usando la curva promedio para el cálculo del $N_{1}$. En estudios dentro del contexto colombiano se recomienda el uso de González [7].

En la Tabla 6 se muestran las correlaciones para la segunda zona geotécnica definida (calle 60), para profundidades entre $(0,30-6,00 \mathrm{~m})$. Se nota una dispersión un poco más alta en relación a la zona I, en comparación con todos los autores, no obstante JRB [21] y Montenegro \& González [8], son las propuestas que más se ajustan al que se considera el valor real obtenido en el laboratorio.

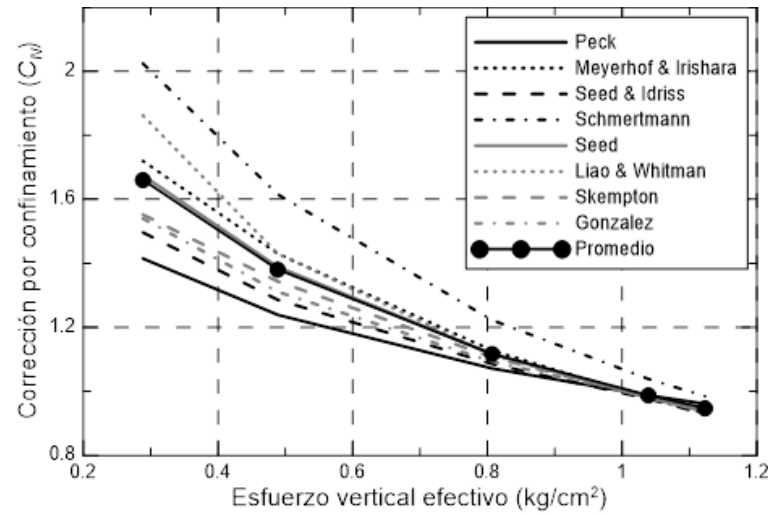

Fig. 6. Corrección por confinamiento para sondeos cercanos a la calle 60 correspondiente a la segunda zona geotécnica. Fuente: Autores

Tabla 6. Correlaciones del ángulo de fricción para la segunda zona geotécnica escogida

$\underline{\left.\text { (Zona II; } \overline{C_{N}}=1,476 ; \mathrm{N}_{1}=16 ; \phi^{\prime} \text { lab }=28^{\circ}\right) \text {. Fuente: Autores }}$

\begin{tabular}{lcc}
\hline \multicolumn{1}{c}{ Autor } & $\phi^{\prime}$ & $\begin{array}{c}\text { Error de disper- } \\
\text { sión teórico }\end{array}$ \\
\hline Terzaghi \& Peck [1] & $32,56^{\circ}$ & $+16,3 \%$ \\
Peck et al. [19] & $32,30^{\circ}$ & $+15,4 \%$ \\
Kishida [20] & $33,02^{\circ}$ & $+17,9 \%$ \\
Schmertmann [16] & $40,44^{\circ}$ & $+44,3 \%$ \\
JNR [21] & $30,04^{\circ}$ & $+7,3 \%$ \\
JRB [21] & $27,34^{\circ}$ & $-2,4 \%$ \\
Hatanaka \& Uchida [22] & $34,25^{\circ}$ & $+22,3 \%$ \\
Montenegro \& González [8] & $28,99^{\circ}$ & $+3.53 \%$ \\
\hline
\end{tabular}

Respecto al SPT-T (Standard Penetration Test + Torque), el cual no posee antecedentes de evaluación en el suelo de la sabana de Bogotá, fue realizado en el campo experimental de la Universidad Católica de Colombia, el cual se encuentra localizado cerca del piedemonte de los cerros orientales, a la altura de la calle 47 .

Fueron realizados ocho sondeos a cinco metros de profundidad cada uno, hallando una similitud con la zona geotécnica II, definida anteriormente, de acuerdo al perfil estratigráfico encontrado, así: 0,00-0,90 $\mathrm{m}$ para rellenos en diferentes tipos de horizontes oscuros con escombros de construcción; 0,90-3,20 m para arcilla café con contenido de limo al final del estrato; 3,20-4,60 $\mathrm{m}$ para limo arenoso gris oscuro con lentes de arena; y 4,60 - final del sondeo para arena fina limosa gris clara. 
En tres de los ochos sondeos fueron realizados ensayos de campo tipo SPT-T, usando un torquímetro Proto Dial Torque Ref. J6121NMF de rango de torque de 35 a $250 \mathrm{lb}-\mathrm{ft}$, con precisión del 4\% de la lectura tomada, como se ve en la Fig. 7.

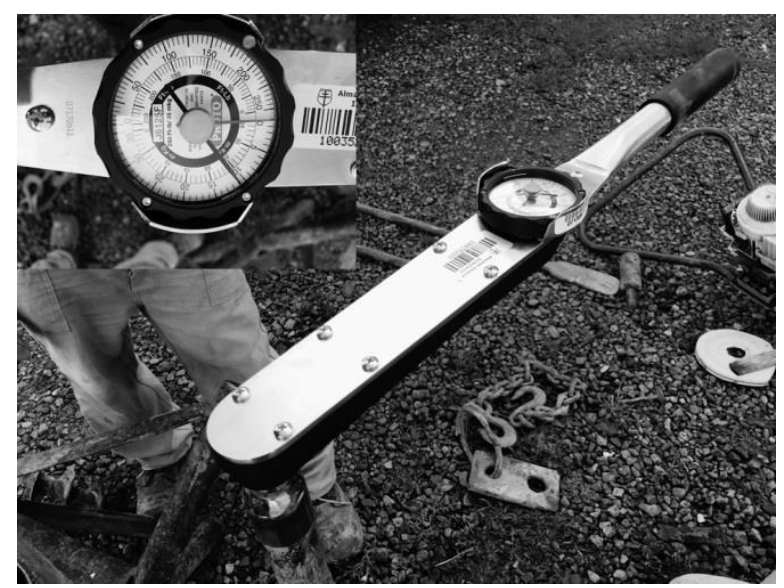

Fig. 7. Torquímetro usado en el SPT-T realizado en el campo experimental de la Universidad Católica de Colombia. Fuente: Autores

En la Tabla 7 se pueden observar los resultados de los SPT-T llevados a cabo, donde la primera parte del ensayo fue basada en el SPT que es regido mediante la norma INV-111-2012, y el torque se realizó considerando la experiencia brasilera en este tipo de ensayos [12]. Se observó especialmente el parámetro $T / N$, el cual según la literatura encontrada, es el factor más importante para correlacionar algunos parámetros geotécnicos como la fricción lateral y capacidad de carga en pilotes [12], así como con fines de clasificación del suelo, que será el ítem considerado en este artículo, ya que este ensayo no presenta antecedentes en Colombia.

De acuerdo con los estudios de Decourt \& Quaresma Filho [25], donde se presentaron correlaciones (1) y (2) entre el torque y el $N 1_{72}$, en suelos sedimentarios y residuales para el contexto brasilero, se observó que a los tres metros de profundidad existe una correlación adecuada entre el número de golpes y el torque aplicado. Para Colombia se utilizó como comparación esta corre- lación con el $N 1_{45}$, de acuerdo con la recomendación de González [7].

$$
\begin{aligned}
& T(k g f \cdot m)=1,10 N_{172} \\
& T(k g f \cdot m)=1,84 N_{1_{72}}
\end{aligned}
$$

Tabla 7. Valores de número del golpes en campo, torque

\begin{tabular}{|c|c|c|c|c|c|}
\hline Profundidad & Sondeo & $\mathrm{N}_{\mathrm{F}}$ & $\begin{array}{c}\mathrm{T}_{\max } \\
\text { (lb-ft) }\end{array}$ & $\begin{array}{c}\mathrm{T}_{\text {res }} \\
(\mathrm{lb}-\mathrm{ft})\end{array}$ & $\begin{array}{c}\mathrm{T} / \mathrm{N} \\
\text { (kg-f.m) }\end{array}$ \\
\hline \multirow{3}{*}{$1,00-1,50 \mathrm{~m}$} & 2 & $4+6$ & 115 & 85 & 1,61 \\
\hline & 5 & $---\cdot$ & $-\cdots--$ & $\cdots-\cdot$ & $-\cdots-$ \\
\hline & 8 & $-\cdots$ & $-\cdots--$ & $\cdots-\cdot$ & $-\cdots$ \\
\hline \multirow{3}{*}{$1,50-2,00 \mathrm{~m}$} & 2 & $4+5$ & 70,0 & 65,0 & 1,09 \\
\hline & 5 & $3+4$ & 82,5 & 60,0 & 0,24 \\
\hline & 8 & $5+5$ & 97,5 & 90,0 & 0,14 \\
\hline \multirow{3}{*}{$2,00-2,50 \mathrm{~m}$} & 2 & $5+6$ & 70,0 & 65,0 & 0,89 \\
\hline & 5 & $3+4$ & 85,0 & 70,0 & 1,70 \\
\hline & 8 & $8+8$ & 60,0 & 52,5 & 0,53 \\
\hline \multirow{3}{*}{$2,50-3,00 \mathrm{~m}$} & 2 & $6+5$ & 80,0 & 55,0 & 1,02 \\
\hline & 5 & $3+3$ & 60,0 & 52,5 & 1,98 \\
\hline & 8 & $10+6$ & 77,5 & 45,0 & 0,68 \\
\hline \multirow{3}{*}{$3,00-3,50 \mathrm{~m}$} & 2 & $1+1$ & 47,5 & 40,0 & 3,33 \\
\hline & 5 & $4+3$ & 67,5 & 65,0 & 1,35 \\
\hline & 8 & $1+1$ & 55,0 & 47,0 & 3,85 \\
\hline \multirow{3}{*}{$3,50-4,00 \mathrm{~m}$} & 2 & $1+1$ & 30,0 & 27,5 & 2,10 \\
\hline & 5 & $2+3$ & 67,5 & 50,0 & 1,89 \\
\hline & 8 & $-\cdots-\cdot$ & -.--- & $-\cdots-\cdot$ & $-\cdots-\cdot$ \\
\hline \multirow{3}{*}{$4,00-4,50 \mathrm{~m}$} & 2 & $1+1$ & N.T. & N.T. & ----- \\
\hline & 5 & $\cdots$ & $-\cdots-$. & $-\cdots-$. & $\cdots-$. \\
\hline & 8 & $-\cdots \cdot$ & $-\cdots-\cdot$ & $\cdots$ & $-\cdots$ \\
\hline
\end{tabular}
máximo y torque residual mediante torquímetro análogo en

A partir de los $3,00 \mathrm{~m}$ se presenta una alta dispersión entre los dos parámetros, debido a presencia de gravillas, que aunque no aumentaron el número de golpes, sí generaron fricción del muestreador incrementando el valor del torque.

Esta relación $T / N$ es útil pues combina la medición de un parámetro estático $(T)$ y uno dinámico $(N)$, además que el torque permite medir la fricción lateral en la región externa del muestreador donde el suelo fue parcialmente alterado, pero conserva la estructura original. De acuerdo con esto, los suelos estructurados tienden a presentar $T / N$ mayores, según Decourt [26] (ver Tabla 8). 
Tabla 8. Clasificación de suelos basada en la relación T/N apud. Fuente: [26]

\begin{tabular}{lcc}
\hline Suelo & $\mathrm{T} / \mathrm{N}$ & Estructura \\
\hline $\begin{array}{l}\text { Arenas sedimentarias capas } \\
\text { inferiores }\end{array}$ & $\approx 0,3$ & Sin estructura \\
$\begin{array}{l}\text { Suelos de cuenca } \\
\text { sedimentaria }\end{array}$ & $\approx 1,2$ & \\
$\begin{array}{l}\text { Suelos saprolíticos } \\
\text { Arcillas porosas colapsables }\end{array}$ & $2,5-5,0$ & \\
$\begin{array}{l}\text { Arcillas blandas } \\
\text { Arenas sedimentarias capas }\end{array}$ & $3,0-4,0$ & \\
inferiores & $\approx 10$ & Estructurado \\
\hline
\end{tabular}

De acuerdo con lo anterior, los suelos de la segunda zona geotécnica definida son poco estructurados, sin embargo, no es adecuado realizar una clasificación del suelo encontrado en el campo experimental a partir del SPT-T, a pesar de que fue desarrollada para un suelo tropical, es necesario avanzar más en la ejecución de este tipo de ensayos en Bogotá, para obtener correlaciones más apropiadas.

\section{CONCLUSIONES}

Se logró zonificar el piedemonte de los cerros orientales en dos regiones afines, de acuerdo con las estratigrafías encontradas en el corredor entre la calle 38 y la calle 170 , encontrando coincidencias con la microzonificación sísmica de Bogotá [15], ya mencionadas al principio del documento.

En el caso de la primera zona conformada por suelos arcillosos con contenidos de limo, donde se pudo analizar la evolución de la corrección por confinamiento propuesta por diferentes autores, se decidió usar el promedio de todos los datos, para el posterior cálculo de la correlación del ángulo de fricción efectivo, sin embargo las propuestas de González [7], Skempton [6] y Seed \& Idriss [4], presentan una tendencia similar al promedio de los datos.

Referente a la correlación del ángulo de fricción, la mayoría de propuestas están dentro del valor significativo del posible error de obtención del mismo, a partir de ensayos de corte directo, sin embargo, la metodología de JRB [21] y Montenegro \& González [8] presentan una dispersión muy alta por defecto. De esta última se explicó anteriormente la posible razón, sin embargo, la correlación es muy sensitiva a la corrección por confinamiento, en especial para valores iniciales muy bajos de $N_{F}$, para valores superiores la correlación tiende a ser menos conservativa (en el evento que el error de dispersión teórico sea por exceso).

Para la segunda zona geotécnica, en estratos ensayados a profundidades más altas la dispersión de datos es muy baja, mientras que cuando se analizan estratos superficiales, la dispersión comienza a aumentar mostrando que la propuesta de Schmertmann [16] se encuentra lejos del promedio por exceso y la de Terzaghi \& Peck [1] por defecto; de nuevo las metodologías de González [7], Skempton [6] y Seed \& Idriss [4], presentan un comportamiento similar al promedio de todos los datos. Al analizar la obtención del ángulo de fricción efectivo mediante las ecuaciones de varios autores, existe una proximidad al calcular este parámetro con todas las ecuaciones, sin embargo, Schmertmann [16] presenta los valores más conservativos y [8]-[21] los menos conservativos, con valores cercanos al real.

Es claro que los ensayos de campo no han alcanzado la tecnología, para que sus variables in situ sean usadas con el fin de correlacionar directamente parámetros geotécnicos de diseño, no obstante para el piedemonte de los cerros orientales, en las correcciones por confinamiento es una buena decisión usar la propuesta de González [7], la cual presenta baja dispersión, al comparar con el promedio del conjunto de datos analizado.

Para la correlación del ángulo de fricción efectivo a partir del $N_{1}$, la mayoría de metodologías presentan proximidad con el valor obtenido de manera real en el laboratorio, sin embargo, no se recomienda usar las metodologías de Schmertmann [16], JRB [21] y Kishida [20] para este cálculo, 
específicamente en los cerros orientales de la ciudad de Bogotá, por la alta dispersión de resultados al comparar con el valor que se considera real (laboratorio), ya que adicional a los factores de seguridad utilizados normalmente en el diseño de una estructura geotécnica, usar metodologías con valores muy conservativos por exceso, terminan aumentando de manera implícita los factores de seguridad, sobrestimando los diseños.

El uso de ensayos in situ SPT-T en Colombia, no tiene antecedentes, por lo que usar este tipo de ensayos para correlacionar parámetros como el ángulo de fricción efectivo es una decisión temeraria, ya que este en principio, en países donde ha sido usado ampliamente, solo se utiliza con fines de clasificación de suelo, cálculo de capacidad de carga de pilotes a fricción y fricción lateral del tándem muestreadorsuelo. Sin embargo, el uso de este tipo de herramientas que complementa el SPT, le puede dar un resurgir a este ensayo muy criticado en nuestro medio, debido a su mala praxis en diferentes condiciones de ejecución.

\section{AGRADECIMIENTOS}

Al Instituto de Desarrollo Urbano por el suministro de la información y a la Universidad Católica de Colombia por los recursos inyectados al proyecto de investigación.

\section{REFERENCIAS}

[1] K. Terzaghi and R. Peck, Soil Mechanics in Engineering Practice. John Wiley and Sons, 1948.

[2] J. E. Bowles, Foundation Analysis and Design. 1988, p. 1004

[3] J. L. Briaud, Geotechnical Engineering: Unsaturated and Saturated Soils, 1st. ed. 2013.

[4] H. B. Seed and I. M. Idriss, "Soil moduli and damping factors for dynamic response analysis," J. Terramechanics, vol. 8, no. 3, p. 109, Jan. 1972.
[5] G. G. Meyerhof, "Discussion on Sand Density by Spoon Penetration," in IV ICSMFE, London, 1957, p. 110.

[6] A. W. Skempton, "Standard penetration test procedures and the effects in sands of overburden pressure, relative density, particle size, ageing and overconsolidation," Géotechnique, vol. 37, no. 3. pp. 411-412, 09-Jan1987.

[7] Á. González, "Estimativos de parámetros efectivos de resistencia con el SPT," in $X$ Jornadas geotécnicas de la ingeniería colombiana, 1999.

[8] L. F. Montenegro, "Calibración del método de parámetros de resistencia con SPT en suelos de la región llanera colombiana," Universidad Nacional de Colombia, sede Bogotá, 2014.

[9] S. M. T. Ranzini, "SPTF," Solos e Rochas, vol. 11, pp. 29-30, 1988.

[10] J. C. Ruge, R. P. da Cunha, and M. Nemocón, "Implementación del ensayo in situ de penetración estándar con torque (SPT-T) y su aplicación en la ingeniería práctica,” 2015.

[11]J. C. Ruge Cárdenas and R. Pinto, "Determinación de parámetros geomecánicos en suelos metaestables, mediante el uso de ensayos de campo," Pre-til, vol. 24, pp. 59-74, 2011.

[12]A. Peixoto, "Estudo do ensaio SPT-T e sua aplicação na prática de engenharia de fundações," Unicamp, 2001.

[13] U. R. Alonso, "Correlação Entre o Atrito lateral medido com o Torque e o SPT," Solos e Rochas, vol. 17, no. 3, pp. 191-194, 1994.

[14] Ingetec, "Estudios y diseños a precio global fijo sin reajustes, de la Troncal Carrera 7 de la Calle 34 a la Calle 170 y tramo de la Calle 72 de la Carrera 7 a Avenida Caracas en Bogotá D.C.," 2006.

[15] FOPAE, "Zonificación de la respuesta sísmica de Bogotá para el diseño sismo resistente de edificaciones," 2010.

[16] J. H. Schmertmann, "Measurement of In-situ Shear Strength," in ASCE Specialty. Conf. on In Situ Measurement of Soil Properties, 1975.

[17] H. Seed, K. Tokimatsu, L. F. Harder, and R. M. Chung, "Influence of SPT Procedures in Soil Liquefaction Resistance Evaluations," J. Geotech. Eng., vol. 111, no. 12, pp. 1425-1445, Dec. 1985.

[18]S. Liao and R. Whitman, "Overburden Correction Factors for SPT in Sand - JGED," ASCE, vol. 112, no. 3, pp. 373-377, 1986.

[19] R. B. Peck, W. E. Hanson, and T. H. Thornburn, Foundation Engineering. John Wiley \& Sons, 1953.

[20] H. Kishida, "Characteristics of liquefied sands during Mino-Owari, Tohnankai and Fukui earthquakes," Soils Found., vol. 9, no. 1, pp. 7592, 1969 . 
[21]JSCE, "Earthquake Resistant Design for Civil Engineering Structures in Japan. Japanese Society of Civil Engineers," Tokyo, 1984.

[22] M. Hatanaka and A. Uchida, "Empirical Correlation between Penetration Resistance and Internal Friction Angle of Sandy Soils," Soils Found., vol. 36, no. 4, pp. 1-9, 1996.

[23] L. Decourt, "SPT, SPT-T: Brazilian practice. Advantages, limitations and criticism. Brazilian Association of Soil Mechanics," 2002.

[24] C. O. Riggs, "North American Standard Penetration Test Practice: An Essay, in Use of in Situ Tests in Geotechnical Engineering," 1986.
[25] L. Decourt and A. R. Quaresma Filho, "Practical Applications of the Standard Penetration Test Complemented by Torque measurements, SPTT; Present Stage and Future Trends," in ICSMFE, XIII. Proc. ISSMGE, 1994, pp. 143146.

[26] L. Decourt, "A More Rational Utilization of Some Old In Situ Tests. In: Geotechnical site characterization," in Proceedings of the first international conference on site characterization, 1998, pp. 913-918. 